\title{
ON THE THEORY OF KILLING ORBITS IN SPACE-TIME
}

\author{
G.S. HALL
}

\begin{abstract}
This paper gives a theoretical discussion of the orbits and isotropies which arise in a space-time which admits a Lie algebra of Killing vector fields. The submanifold structure of the orbits is explored together with their induced Killing vector structure. A general decomposition of a space-time in terms of the nature and dimension of its orbits is given and the concept of stability and instability for orbits introduced. A general relation is shown linking the dimensions of the Killing algebra, the orbits and the isotropies. The well-behaved nature of "stable" orbits and the possible miss-behaviour of the "unstable" ones is pointed out and, in particular, the fact that independent Killing vector fields in space-time may not induce independent such vector fields on unstable orbits. Several examples are presented to exhibit these features. Finally, an appendix is given which revisits and attempts to clarify the well-known theorem of Fubini on the dimension of Killing orbits.
\end{abstract}

\section{INTRODUCTION}

The purpose of this paper is to develop in a reasonably modern way the theory of Killing orbits in space-times. It is not claimed that all the results given are new but, at least in the form presented here, some are new to the present author. It is hoped that the proofs given are a little tidier and that the conditions required for the results stated are clearer.

The theory of Killing symmetry is often used in the construction of exact solutions of Einstein's field equations and is important in the physical applications of general relativity. However, certain mathematical problems arise in the study of such symmetries and it is of some importance for these to be at least realised and, if possible, rectified. For example, exact solutions are often found by imposing a certain collection of Killing vector fields on a space-time with orbits of a certain prescribed nature (timelike, spacelike or null) and dimension. The resulting metrics, not surprisingly, have precisely these symmetries and orbits. However, they may be extendible to larger manifolds where the orbit nature and dimension could change. A less restrictive way of approaching such a problem is to find general results which link the possible orbit types with the dimension of the Killing algebra. This will be discussed in more detail later in the paper. Another potential problem arises from what the author perceives as the occasional unjustified (unconscious) assumption that independent Killing vector fields in space-time naturally project to independent Killing vector fields in the induced geometry of any (non-null) orbit and thus to physical interpretations in this orbit geometry. That the Killing nature is preserved is clear, but the independence need not be. Examples will be given later where this independence assumption fails. It will also be shown that this assumption is correct provided a type of "stability" condition is imposed on the orbit and that this condition naturally holds for the orbits through "almost every" point of the space-time. This stability property of orbits has some consequences for the nature of the Killing isotropy at a space-time point on the orbit. Such isotropy is important for the physical interpretation of the solution and may depend on the stability of the orbit. This will also be clarified later. It would be interesting to find a general physical interpretation of metrics which admit unstable orbits. There 
are also some mathematical problems associated with the orbits arising naturally from the imposed algebra of Killing vector fields and these will also be reviewed. It is, after all, important from a physical viewpoint, that such orbits are sufficiently well behaved for calculus to be used on them. A further problem occurs with the well-known application of Fubini's theorem to the possible dimension of the Killing algebra. This is discussed and resolved in the appendix.

Throughout the paper $M$ will denote the usual smooth (connected, Hausdorff, 4-dimensional) space-time manifold with smooth Lorentz metric $g$ of signature $(-,+,+,+)$. Thus $M$ is paracompact. A comma, semi-colon and the symbol $\mathcal{L}$ denote the usual partial, covariant and Lie derivative, respectively, the covariant derivative being with respect to the Levi-Civita connection $\Gamma$ on $M$ derived from $g$. The associated curvature, Weyl and Ricci tensors will be denoted in component form by $R_{b c d}^{a}, C^{a} b c d$ and $R_{a b}\left(\equiv R_{b c d}^{c}\right)$. If $m \in M$, the tangent space to $M$ at $m$ is denoted by $T_{m} M$. A 2-dimensional subspace $U$ of $T_{m} M$ is referred to as a 2-space and is called spacelike if each non-zero member of $U$ is spacelike, null if it contains exactly one null direction and timelike if it contains exactly two distinct null directions. Such a classification is mutually exclusive and exhaustive of 2 -spaces at any $m \in M$. It follows that $U$ is spacelike if and only if it contains no null members and that $U$ is timelike if and only if it contains a timelike member. The family of 3-dimensional subspace (3-spaces) of $T_{m} M$ can be similarly classified as spacelike, null and timelike, the definitions for spacelike and null 3-spaces being identical to those for 2-spaces. A 3-space $U$ is called timelike if it contains at least two (and hence infinitely many) distinct null directions and this is equivalent to it containing a timelike member. A non-zero skew-symmetric tensor (bivector) $F$ at $m$ has even (matrix) rank. If its rank is two it is called simple and may be written as $F_{a b}=2 p_{[a} q_{b]}$ for covectors $p$ and $q$ at $m$. The 2 -space at $m$ spanned by $p^{a}$ and $q^{a}$ is called the blade of $F$ and $F$ is called spacelike (respectively, timelike, null) if this blade is spacelike (respectively, timelike, null). If $F$ has rank four it is called non-simple.

\section{Submanifolds and Generalised Distributions on $M$}

In this section only, $M$ can be any (smooth) paracompact manifold. A subset $N$ of $M$ is called a submanifold of $M$ if $N$ itself is a manifold and if the inclusion map $i: N \rightarrow M$ is a smooth immersion (i.e. the derived linear map $i_{*}$ has rank equal to $\operatorname{dim} N$ at each $m \in M$, or equivalently, $i_{*}$ is a one-to-one map $T_{m} N \rightarrow T_{m} M$ at each $m \in N)$. The natural manifold topology of $N$ need not equal the subspace topology on $N$ induced from the manifold topology of $M$ (but always contains it since $i$ is continuous). If it does, $N$ is called a regular submanifold of $M[1$. It is remarked here that any topological property given to a submanifold $N$ refers to its natural manifold topology and may not necessarily hold with respect to its induced subspace topology from $M$ unless $N$ is regular. It is also noted that an open subset of $M$ has a natural structure as an open submanifold of $M$ of the same dimension as $M$ and is regular. It is clear that if $N^{\prime}$ is a submanifold of $N$ and $N$ is a submanifold of $M$ then $N^{\prime}$ is a submanifold of $M$. Less obvious is the fact that if $N^{\prime}$ and $N$ are submanifolds of $M$, with $N$ regular, and if $N^{\prime} \subseteq N$, then $N^{\prime}$ is a submanifold of $N$. A subset $N$ of $M$ may be given more than one structure as a submanifold of $M$ and these structures may have different dimensions. Unfortunately, it is not convenient simply to insist in the definition of a submanifold that it is regular since many 'natural submanifolds' evolve in differential geometry which are not regular. Regular submanifolds have some pleasant properties which do not necessarily hold for non-regular submanifolds. For example, if $M_{1}$ and $M_{2}$ are any smooth manifolds and if $f: M_{1} \rightarrow M_{2}$ is a smooth map whose range $f\left(M_{1}\right)$ lies inside a submanifold 
$N$ of $M_{2}$, then $f$, considered as a map $f: M_{1} \rightarrow N$, is not necessarily smooth, or even continuous. (In fact, if it is continuous, it is smooth.) However, if $N$ is a regular submanifold of $M_{2}$ then the map $f: M_{1} \rightarrow N$ is necessarily smooth. It should be noted, however, that if a subset $N$ of $M$ can be given the structure of a regular submanifold of $M$ then its dimension is fixed (by $M$ and $N$ ) and it admits no other submanifold structures of this dimension. Thus such a regular submanifold structure on $N$ is unique. To see this note that if $N_{1}$ and $N_{2}$ denote the subset $N$ with two regular submanifold structures, the smooth inclusion map $i: N \rightarrow M$ leads to a smooth bijective map $i: N_{1} \rightarrow N_{2}$ with smooth inverse and so $N_{1}$ and $N_{2}$ are diffeomorphic. A standard property of immersions can then be used [1] to show that if $N_{3}$ is any submanifold structure on $N$ of the same dimension as $N_{1}$ then $N_{3}$ coincides with $N_{1}$.

There is structure which is intermediate between submanifolds and regular submanifolds. A subset $N$ of $M$ is called a leaf [2] if it is a connected submanifold of $M$ and if whenever $T$ is any locally connected topological space and $f: T \rightarrow M$ a continuous map with $F(T) \subseteq N$, the map $f: T \rightarrow N$ is continuous. It follows that if $M_{1}$ and $M_{2}$ are any paracompact manifolds and $N \subseteq M_{2}$ is a leaf and if $f: M_{1} \rightarrow M_{2}$ is a smooth map whose range $f\left(M_{1}\right)$ lies in $N$ then the map $f: M_{1} \rightarrow N$ is continuous and hence smooth [2]. It follows by an argument similar to that for regular submanifolds that if $N$ can be given the structure of a leaf of $M$ then its dimension is fixed (by $M$ and $N$ ) and that it admits no other submanifold structure of this dimension. Thus such a leaf structure on $N$ is unique.

It is clear that every connected regular submanifold of $M$ is a leaf of $M$ and, of course, that every leaf of $M$ is a connected submanifold of $M$. Neither converse is true. In fact the well known 'irrational wrap' on the torus is a leaf which is not regular [2] and the 'figure of eight' (connected) submanifold in $\mathbb{R}^{2}$ [1] is not a leaf.

Another useful result is now available. Let $M_{1}$ and $M_{2}$ be (smooth) manifolds with respective submanifolds $N_{1}$ and $N_{2}$ and let $f: M_{1} \rightarrow M_{2}$ be a smooth map such that $f\left(N_{1}\right) \subseteq N_{2}$. Then the associated map $f: N_{1} \rightarrow M_{2}$ is clearly also smooth (since it involves only an elementary composition with the smooth immersion $i_{1}$ : $N_{1} \rightarrow M_{1}$ ) and if the associated map $f: N_{1} \rightarrow N_{2}$ is also smooth then the differential of $f, f_{*}$, maps the tangent space $T_{m} N_{1}$ to $N_{1}$ at $m$ into the tangent space $T_{f(m)} N_{2}$ to $N_{2}$ at $f(m)$ [1. A special case of this result will be useful in what is to follow. This is that if $M$ is a smooth paracompact manifold and $N$ is a leaf of $M$ and if $f: M \rightarrow M$ is smooth and $f(N) \subseteq N$, then $f$ is smooth and $f_{*}$ maps $T_{m} N$ into $T_{f(m)} N$ for $m \in N$. If, in addition, $\mathrm{f}$ is a diffeomorphism then $f_{*}$ is an isomorphism $T_{m} N \rightarrow T_{f(m)} N$ at each $m \in N$.

Now let $A$ be a vector space of global smooth vector fields on $M$. Define a generalised distribution $\Delta$ on $M$ as a map which associates with each $m \in M$ a subspace $\Delta(m)$ of $T_{m} M$ given by

$$
m \rightarrow \Delta(m)=\{X(m): X \in A\} .
$$

There is no requirement that $\operatorname{dim} \Delta(m)$ be constant on $M$ and so $\Delta$ is not necessarily a Fröbenius type of distribution.

For any global smooth vector field $X$ on $M$ there is associated a collection of local smooth diffeomorphisms (local 'flows') on $M$ each of which is obtained in the following way. Let $m^{\prime} \in M$. Then there exists an open neighbourhood $U$ of $m^{\prime}$ and a real number $\epsilon>0$ such that through any point $m$ of $U$ there is an integral curve $c_{m}$ of $X$ defined on $(-\epsilon, \epsilon)$ such that $c_{m}(0)=m$. The maps $\phi_{t}: U \rightarrow M$ defined by $\phi_{t}(m)=c_{m}(t)(t \in(-\epsilon, \epsilon))$ are the required local smooth diffeomorphisms [3] 4 . The set of all such local diffeomorphisms obtained in this way for each $m^{\prime} \in M$ and each pair $(U, \epsilon)$ is the required collection. 
Now let $A$ be a Lie algebra of global smooth vector fields on $M$ under the usual Lie bracket operation. Let $X_{1}, \ldots X_{k} \in A$ and $\phi_{t_{1}}^{1} \ldots \phi_{t_{k}}^{k}$ be the local flows associated with them, for appropriate values of $t_{1}, \ldots t_{k}$. Consider the set of all local diffeomorphisms (where defined) of the form

$$
m \rightarrow \phi_{t_{1}}^{1}\left(\phi_{t_{2}}^{2}\left(\ldots \phi_{t_{k}}^{k}(m) \ldots\right)\right) \quad m \in M
$$

for each choice of $k \in \mathbb{N}, X_{1}, \ldots X_{k} \in A$ and admissible $\left(t_{1}, \ldots, t_{k}\right) \in \mathbb{R}^{k}$. Now define a relation $\sim$ on $M$ by $m_{1} \sim m_{2}$ if and only if some local diffeomorphism of the form (2) maps $m_{1}$ into $m_{2}$. The relation $\sim$ is, in fact, an equivalence relation [2, 5] and the associated equivalence classes are called the orbits of A. Further, each of these orbits can be given a unique structure as a connected submanifold of $M$ [2, 5] and is then a leaf of $M$ 2. The interesting question arises as to whether $A$ is integrable, that is, whether the orbits are integral manifolds of $A$. More precisely, for each $m \in M$, does $\Delta(m)$, if non-trivial, coincide with the subspace of $T_{m} M$ tangent to the orbit of A? Without some further restriction on $A$ the answer is easily shown to be no. Suppose now that $A$ is a Lie algebra of smooth vector fields on $M$ under the Lie bracket operation. If, in addition, $A$ leads to a Fröbenius type of distribution, so that $\operatorname{dim} \Delta(m)$ is constant on $M$, the integrability of $A$ follows from Fröbenius' theorem (see, e.g. 1]). However, without the constancy of $\operatorname{dim} \Delta(m)$ the result may fail. To see this the following modified version of an example in 5 suffices. Let $M=\mathbb{R}^{2}$ with the usual global $x, y$ coordinate system and let $f: \mathbb{R}^{2} \rightarrow \mathbb{R}$ be the global smooth function given by $f(x, y)=0(x \leq 0), f(x, y)=e^{-\frac{1}{x}}(x>0)$. Then define vector fields $X_{k}(k \in \mathbb{N})$ on $M$ by

$$
X_{1}=\frac{\partial}{\partial x}, X_{2}=f \frac{\partial}{\partial y}, \ldots, X_{k}=\frac{\partial^{k-2} f}{\partial x^{k-2}} \frac{\partial}{\partial y}, \ldots
$$

Let $A$ be the vector space spanned by the vector fields in (3). Then $A$ is a Lie algebra since $\left[X_{1}, X_{k}\right]=X_{k+1}(k \geq 2)$ and $\left[X_{p}, X_{q}\right]=0(p, q \geq 2)$. Since the only solution of the equation $\sum_{k=0}^{n} a_{k} \frac{\partial^{k} f}{\partial x^{k}}=0$ for $a_{0}, \ldots, a_{k} \in \mathbb{R}$ and for all $x \in \mathbb{R}, x>0$, is $a_{0}=a_{1}=\ldots=a_{k}=0, A$ is infinite-dimensional. Also, $\operatorname{dim} \Delta(m)=2$ in the open region $x>0$ of $M$ and $\operatorname{dim} \Delta(m)=1$ if $x \leq 0$. However, from (2) and (3) it can be seen that $M$ is the only orbit and has dimension two. Thus $A$ is a Lie algebra but fails to be integrable.

In the above example $A$ was infinite-dimensional. The general problem of finding integrability conditions for $A$ when $\operatorname{dim} \Delta(m)$ is not constant over $M$ (i.e. the generalisation of Fröbenius' theorem) has been extensively discussed. The following result is a consequence of a general result due to Hermann [6 but draws also on the work of Sussmann [5] and Stefan [2, 7].

Theorem 1. Let $A$ be a finite-dimensional Lie algebra of global smooth vector fields on a paracompact smooth manifold $M$ with associated generalised distribution $\Delta$. If $m \in M$ and $\operatorname{dim} \Delta(m)=0$ the orbit through $m$ is $\{m\}$. If $\operatorname{dim} \Delta(m)>0$ the orbit through $m$ is a leaf of $M$ and $A$ is integrable in that at each such $m \in M, \Delta(m)$ coincides with the subspace of $T_{m} M$ tangent to the orbit through $m$.

\section{Lie algebras of Vector Fields on Space-Times}

Now let $M$ be a space-time. A submanifold $N$ of $M$ of dimension 1,2 or 3 is said to be timelike (respectively, spacelike or null) at $m \in N$ if the subspace of $T_{m} M$ tangent to $N$ is timelike (respectively, spacelike or null). Also $N$ is called timelike (respectively, spacelike or null) if it is timelike (respectively, spacelike or null) at each $m \in N$.

Now let $A$ be a vector space of global smooth vector fields on $M$ with associated generalised distribution $\Delta$. In the study of the set $A$ it is convenient to know the 
variation of the type and dimension of $\Delta(m)$ over $M$. Thus for $i=0,1,2,3,4$ define the subsets $V_{i}$ of $M$ by

$$
V_{i}=\{m \in M: \operatorname{dim} \Delta(m)=i\}
$$

and then for $p=1,2,3$ define the subsets $S_{p}, T_{p}$ and $N_{p}$ of $M$ by

$$
\begin{aligned}
S_{p}=\{m \in M: \operatorname{dim} \Delta(m)=p & \text { and } \Delta(m) \text { is spacelike }\} \\
T_{p}=\{m \in M: \operatorname{dim} \Delta(m)=p & \text { and } \Delta(m) \text { is timelike }\} \\
N_{p}=\{m \in M: \operatorname{dim} \Delta(m)=p & \text { and } \Delta(m) \text { is null }\}
\end{aligned}
$$

Thus $M=\bigcup_{i=0}^{4} V_{i}$ and $V_{p}=S_{p} \cup T_{p} \cup N_{p}$ for $p=1,2,3$. The following result gives two ways of disjointly decomposing $M$ in which, for a subset $U$ of $M$, int $U$ means the topological interior of $U$ with respect to the manifold topology on $M$. It tidies up the work in 8 where there were careless slips (see 9] for a corrected version). The following version of the rank theorem will also be used. If for $m^{\prime} \in M$ $\operatorname{dim} \Delta\left(m^{\prime}\right)=k\left(1 \leq k \leq 4\right.$ then there exists an open neighbourhood of $m^{\prime}$ at each point of which $\operatorname{dim} \Delta(m) \geq k$.

Theorem 2. Let $M$ be a space-time and $A$ a vector space of global smooth vector fields on $M$. Then, with the above notation, $M$ may be directly decomposed in the following ways.

$$
\begin{gathered}
M=\bigcup_{i=0}^{4} \operatorname{int} V_{i} \cup Z_{1}=V_{4} \cup \bigcup_{i=0}^{3} \operatorname{int} V_{i} \cup Z_{1} \\
M=V_{4} \cup \bigcup_{p=1}^{3} \operatorname{int} S_{p} \cup \bigcup_{p=1}^{3} \operatorname{int} T_{p} \cup \bigcup_{p=1}^{3} \operatorname{int} N_{p} \cup \operatorname{int} V_{0} \cup Z
\end{gathered}
$$

where $Z_{1}$ and $Z$ are closed subsets of $M$ defined by the disjointness of the decompositions and which have empty interior, $\operatorname{int} Z_{1}=\operatorname{int} Z=\emptyset$.

Proof. For the first decomposition the rank theorem shows that $V_{4}$ is open and hence that $V_{4}=\operatorname{int} V_{4}$. The same theorem also reveals that the subsets $M_{n}=$ $\bigcup_{i=n}^{4} V_{i}(0 \leq n \leq 4)$ are open in $M$. Now, by the disjointness of (6), $Z_{1}$ is closed in $M$ and so let $W \subseteq Z_{1}$ be an open subset of $M$. By disjointness, $W \cap V_{4}=\emptyset$. Then $W \cap M_{3}$ is open and equals $W \cap V_{3}$. If this latter subset is not empty then, since it is open, it implies that $W \cap \operatorname{int} V_{3} \neq \emptyset$. This contradicts the disjointness of the decomposition (6) and so $W \cap V_{3}=\emptyset$. Similarly $W \cap M_{2}=W \cap V_{2}$ and is open and it follows that $W \cap V_{2}=\emptyset$. Repeating the argument leads to $W \cap V_{i}=\emptyset(0 \leq i \leq 4)$ and hence $W=\emptyset$. Thus $\operatorname{int} Z_{1}=\emptyset$.

For the second decomposition it is again a matter of checking that the (closed) subset $Z$ has empty interior. So let $W \subseteq Z$ be open. As above, $W \cap V_{4}=\emptyset$ and the subset $\widetilde{W}$ given by

$$
\widetilde{W} \equiv W \cap M_{3}=W \cap V_{3}=W \cap\left(S_{3} \cup T_{3} \cup N_{3}\right)
$$

is open. Suppose $\widetilde{W} \neq \emptyset$. If $W$ is disjoint from $S_{3}$ and $T_{3}$ one has $W \cap N_{3}$ open and non-empty. But this implies that $W \cap \operatorname{int} N_{3} \neq \emptyset$ and contradicts the disjointness of the decomposition (7). So $W$ intersects $S_{3} \cup T_{3}$ non-trivially. If $W \cap S_{3} \neq \emptyset$ let $p \in W \cap S_{3} \subseteq \widetilde{W}$. Now $\operatorname{dim} \Delta(m)=3$ at each $m \in \widetilde{W}$ and so there exists $X, Y, Z \in A$ such that $X(p), Y(p)$ and $Z(p)$ span the spacelike 3 -space $\Delta(p)$ at $p$. But this implies that the normal direction to $\Delta(p)$ is timelike. If $u \in T_{p} M$ spans this direction then, in some coordinate neighbourhood $U \subseteq \widetilde{W}$ of $p$ the components $X^{a}, Y^{a}, Z^{a}$ and $u^{a}$ satisfy, at $p$, the equations

$$
X_{a} u^{a}=0, Y_{a} u^{a}=0, Z_{a} u^{a}=0 .
$$


Suppose, without loss of generality, that the last three columns of the left hand sides of the array (9) form a non-singular $3 \times 3$ system for $u^{1}, u^{2}$ and $u^{3}$ at $p$. Then, together with a fixed (constant) choice of $u^{0}$, Cramer's rule shows that (9) can be solved for $u^{a}$ uniquely at $p$ and in some open neighbourhood $U^{\prime} \subseteq U$ of $p$ at each point of which $X, Y$ and $Z$ are independent tangent vectors. The components $u^{1}, u^{2}, u^{3}$ and $u^{0}$ are then smooth functions on $U^{\prime}$ and hence give rise to a timelike vector field (also called u) in some open neighbourhood $U^{\prime \prime}(\subseteq \widetilde{W})$ of $p$. It follows that at each point of $U^{\prime \prime}, \Delta(m)$ is 3-dimensional (since $U^{\prime \prime} \subseteq \widetilde{W}$ ) and spacelike (since it is orthogonal to $u$ at each such point). Hence $p \in U^{\prime \prime} \subseteq S_{3}$ and so $U^{\prime \prime} \subseteq \operatorname{int} S_{3}$. This gives the contradiction $W \cap$ int $S_{3} \neq \emptyset$ to the disjointness of the decomposition and so $W \cap S_{3}=\emptyset$. Similarly one can establish that $W \cap T_{3}=\emptyset$ and the contradiction that $W$ is disjoint from $S_{3}$ and $T_{3}$. It follows that $\widetilde{W}=W \cap M_{3}=\emptyset$. One then repeats the argument on the open subsets $W \cap M_{2}$ and then $W \cap M_{1}$ to finally achieve $W=\emptyset$ and the conclusion that int $Z=\emptyset$.

\section{Killing Symmetry in Space-Times}

Let $M$ be a space-time and let $K(M)$ be the vector space of all global smooth Killing vector fields on $M$ and with associated generalised distribution $\Delta$. Then if $X \in K(M)$ one has $\mathcal{L}_{X} g=0$ and thus in any coordinate domain in $M$

$$
\begin{gathered}
X_{a ; b}+X_{b ; a}=0\left(\Leftrightarrow X_{a ; b}=F_{a b}=-F_{b a}\right) \\
X_{; b c}^{a}=F_{b ; c}^{a}=R_{b c d}^{a} X^{d}
\end{gathered}
$$

where $F$ is the Killing bivector of $X$. It easily follows that (10) and (11) give rise along any curve in $M$ to a system of first order differential equations in the ten quantities $X^{a}$ and $F_{a b}$. Since $M$ is path connected each $X \in K(M)$ is uniquely determined by the values of $X$ and $F$ at some (any) point of $M$ (and so $\operatorname{dim} K(M) \leq$ 10). Hence if $X$ vanishes on some non-empty open subset of $M, X \equiv 0$ on $M$ (and thus int $V_{0}=\emptyset$ in (6) and (7) if $A \equiv K(M)$ is non-trivial). It is also well known that $X, Y \in K(M) \Rightarrow[X, Y] \in K(M)$ and so $K(M)$ is a finite-dimensional Lie algebra called the Killing algebra of $M$. It follows from theorem 1 that $K(M)$ is integrable so that, in the sense of that theorem, the orbit structure is well behaved.

Now suppose $M$ is decomposed as in (4)-(7) with $K(M)$ playing the role of $A$ in these equations. First consider the subset $V_{4}$ of $M$ and suppose $V_{4} \neq \emptyset$. Then $V_{4}$ is an open submanifold of $M$ with Lorentz metric induced from the metric $g$ on $M$. Let $K\left(V_{4}\right)$ be the restriction to $V_{4}$ of the members of the Killing algebra $K(M)$. The generalised distribution on $V_{4}$ associated with $K\left(V_{4}\right)$ has dimension four at each point and so from Chow's theorem 10 (see also [2, 5]) the associated orbits of $K\left(V_{4}\right)$ are precisely the components of $V_{4}$. Hence the orbits of $K(M)$ through any point of $V_{4}$ are the components of $V_{4}$.

Now let $O$ be a proper orbit of $K(M)$ (i.e. $1 \leq \operatorname{dim} O \leq 3)$. Then, from theorem $1, O$ is a leaf of $M$. Now let $f$ be any local diffeomorphism of the type (2) arising from $K(M)$ and with domain the open subset $U$ of $M$ and range the open subset $U^{\prime} \equiv f(U)$ of $M$. Suppose $U \cap O \neq \emptyset$. Then $f: U \rightarrow M$ is smooth and since $O \cap U$ is an (open) submanifold of $O$ and hence of $U, f$ restricts to a smooth map $O \cap U \rightarrow M$ whose range lies in the leaf $O$ of $M$. Thus (section 2) it restricts to a smooth map $O \cap U \rightarrow O$ and hence to a smooth map $f: O \cap U \rightarrow O \cap U^{\prime}$ since $O \cap U^{\prime}$ is an open and hence regular manifold of $O$. Thus, in this sense, each such map $f$ restricts to a local diffeomorphism of $O$. It now follows (section 2 ) that if $m \in O \cap U$ and $m^{\prime} \equiv f(m) \in O \cap U^{\prime}$ and if $u, v \in T_{p} M$ are each tangent to $O$ at $m$ then $f_{*} u$ and $f_{*} v$ are tangent to $O$ at $m^{\prime}$. Since the statement that $X$ is a 
Killing vector field on $M$ is equivalent to each local flow $\phi_{t}$ of $X$ satisfying the local isometry condition $\phi_{t}^{*} g=g$, where $\phi_{t}^{*}$ is the usual pullback map, one has

$$
g_{m^{\prime}}\left(f_{*} u, f_{*} v\right)=\left(f^{-1 *} g\right)_{m^{\prime}}\left(f_{*} u, f_{*} v\right)=g_{m}(u, v)
$$

where $g_{m}=g(m)$. From this it easily follows from the definitions of section 1 that the nature (spacelike, timelike or null) of $O$ is the same at each point of $O$.

Now suppose that $O$ is proper and non-null and let $i: O \rightarrow M$ be the inclusion map. Since $O$ is connected (theorem 1 ) it easily follows from the previous paragraph that $O$ is either spacelike or timelike. Then $g$ induces a metric $h \equiv i^{*} g$ on $O$ which is a positive definite metric if $O$ is spacelike and a Lorentz metric if $O$ is timelike. Since $K(M)$ is integrable, any $X \in K(M)$ is tangent to $O$ and projects naturally to a smooth global vector field $\widetilde{X}$ on $O$ such that $i_{*} \widetilde{X}=X$ on $O$ [1]. Also if $\phi_{t}$ is a local flow (isometry) of $X$ and $\widetilde{\phi}_{t}$ the corresponding local flow on $O$ associated with $\widetilde{X}$ then $i \circ \widetilde{\phi}_{t}=\phi_{t} \circ i$. To see this note that if $\widetilde{c}$ is an integral curve of $\tilde{X}$ then $c \equiv i \circ \widetilde{c}$ is an integral curve of $X$ 1]. Conversely, if $c$ is an integral curve of $X$ and if $\widetilde{c}$ is defined by $c=i \circ \widetilde{c}$ then $\widetilde{c}$ is an integral curve of $\widetilde{X}$. This follows because $c: I \rightarrow M$ is smooth for some open interval $I$ of $\mathbb{R}$ and its range lies in the leaf $O$ and is represented by the map $\widetilde{c}$, which is thus smooth. The condition that $c$ is an integral curve of $X$ is $X \circ c=c_{*} \circ \frac{\partial}{\partial t}$ (with $t$ the parameter of $c$ ) and so $c_{*} \circ \frac{\partial}{\partial t}=(\underline{i} \circ \widetilde{c})_{*} \circ \frac{\partial}{\partial t}=i_{*} \circ \widetilde{c}_{*} \circ \frac{\partial}{\partial t}=X \circ c=X \circ i \circ \widetilde{c}=i_{*} \circ \widetilde{X} \circ \widetilde{c}$. Since $i$ is an immersion (and so $i_{*}$ is injective), $\widetilde{c}_{*} \circ \frac{\partial}{\partial t}=\widetilde{X} \circ \tilde{c}$ and so $\tilde{c}$ is an integral curve of $\widetilde{X}$. Now since $\phi_{t}^{*} g=g$ one has

$$
\widetilde{\phi}_{t}^{*} h=\widetilde{\phi}_{t}^{*}\left(i^{*} g\right)=\left(i \circ \widetilde{\phi}_{t}\right)^{*} g=\left(\phi_{t} \circ i\right)^{*} g=i^{*}\left(\phi_{t}^{*} g\right)=h
$$

and so $\widetilde{X}$ is a Killing vector field on $O$ with respect to $h$. Also, if $X, Y \in K(M)$ with associated vector fields $\widetilde{X}$ and $\widetilde{Y}$ on $O$ a standard result on Lie brackets gives $i_{*}[\widetilde{X}, \widetilde{Y}]=[X, Y]$. It follows that the map $k: X \rightarrow \widetilde{X}$ is a Lie algebra homomorphism $K(M) \rightarrow K(O)$ where $K(O)$ is the Lie algebra of global smooth Killing vector fields on $O$.

The linear map $k$ need not be either injective or surjective. To see that it is not necessarily surjective consider the space-time $M=\left\{(t, x, y, z) \in \mathbb{R}^{4}, t>0\right\}$ with metric given by

$$
d s^{2}=-d t^{2}+t d x^{2}+e^{2 t} d y^{2}+e^{3 t} d z^{2}
$$

Here $K(M)$ is 3 -dimensional being spanned by $\frac{\partial}{\partial x}, \frac{\partial}{\partial y}$ and $\frac{\partial}{\partial z}$. Any orbit $O$ of $K(M)$, however, is a 3-dimensional spacelike hypersurface of constant $t$ which, with its induced metric, is 3-dimensional Euclidean space. Hence $\operatorname{dim} K(O)=6$.

To see that $k$ need not be injective one first notes that lack of injectivity would require $X \in K(M), X \not \equiv 0$, with $\widetilde{X} \equiv 0$ on $O$. This means that $X$ should vanish on $O$. To investigate this possibility let $X \in K(M), X \not \equiv 0$ be such that $X(m)=0$ for some $m \in M$. The associated local flows $\phi_{t}$ associated with $X$ then satisfy $\phi_{t}(m)=m$ and $m$ is called a zero (or a fixed point) of $X$. Let $U$ be a coordinate neighbourhood of $m$ with coordinates $y^{a}$. The linear isomorphisms $\phi_{t *}: T_{m} M \rightarrow T_{m} M$ are represented in the basis $\left(\frac{\partial}{\partial y^{a}}\right)_{m}$ by the matrix (or its transpose depending on notation)

$$
e^{t B}=\exp t\left(B_{b}^{a}\right), B_{b}^{a}=\left(\frac{\partial X^{a}}{\partial y^{b}}\right)_{m}
$$

where $B$ is the linearisation of $X$ at $m$. Since $X \in K(M)$ it follows from (10) that $B_{b}^{a}=F_{b}^{a}(m)$. Now $X$ is an affine vector field and so if $\chi$ is the usual exponential diffeomorphism from some open neighbouhood of $o \in T_{m} M$ onto some 
open neighbourhood $V$ of $m$ then 3

$$
\phi_{t} \circ \chi=\chi \circ \phi_{t *}
$$

In the resulting normal coordinate system on $V$ with coordinates $x^{a}$ it follows that one has the convenient situation that the components $X^{a}$ of $X$ are linear functions of the $x^{a}, X^{a}=B_{b}^{a} x^{b}$ (see, e.g. [11, 12]). Since $B_{b}^{a}\left(=F_{b}^{a}(m)\right)$ is skew self-adjoint with respect to $g(m)$ (i.e. $B_{a b} \equiv g_{a c} B_{b}^{c}=-B_{b a}$ at $m$ ) the rank of $B$ is even. If $B=0$ then a remark at the beginning of this section shows that $X \equiv 0$ on $M$. Thus the rank of $B$ is two or four. The zeros of $X$ in $V$ have coordinates satisfying $B_{b}^{a} x^{b}=0$ and so, if $\operatorname{rank} B=4$, the zero $m$ of $X$ is the only zero in $V$ (and so is isolated). If, however, $\operatorname{rank} B=2$ the set of zeros of $X$ in $V$ can be given the structure of a 2-dimensional regular manifold $N$ of the open submanifold $V$ of $M$ 11, 12. Thus if $k$ is not injective with $X$ vanishing on the orbit $O$ through $m$, the zeros of $X$ are not isolated since, in $V$, they are precisely the points of $N$. The next step is to get some information on the dimension of $O$ using the discussion in section 2. To do this let $O^{\prime}=O \cap V$ so that $O^{\prime}$ is an open subset, and hence an open submanifold, of $O$ and hence a submanifold of $M$. Then $O^{\prime}$ is a submanifold of $M$ contained in the regular submanifold $V$ of $M$ and is then a submanifold of $V$. By the definition of $N$ it then follows that $O^{\prime} \subseteq N \subseteq V$ with $O^{\prime}$ and $N$ submanifolds of $V$ with $N$ regular. From this it follows that $O^{\prime}$ is a submanifold of $N$ and so $\operatorname{dim} O^{\prime} \leq \operatorname{dim} N$. This finally gives $\operatorname{dim} O\left(=\operatorname{dim} O^{\prime}\right) \leq 2$. Thus if $\operatorname{dim} O=3, k$ is injective.

The above results can now be collected together.

Theorem 3. Let $M$ be a space-time and let $O$ be an orbit of $K(M)$.

(i) If $\operatorname{dim} O=4, O$ is a component of $V_{4}$ in the decomposition (6) and the map $k: K(M) \rightarrow K(O)$ is an injective Lie algebra homomorphism.

(ii) If $1 \leq \operatorname{dim} O \leq 3$ then $O$ is spacelike, timelike or null. If, in addition, $O$ is not null, the map $K: K(M) \rightarrow K(O)$ is a Lie algebra homomorphism which is not necessarily surjective. It is injective if $\operatorname{dim} O=3$ but need not be if $\operatorname{dim} O=1$ or 2 . If $k$ is injective then $K(M)$ is Lie-isomorphic to a Lie subalgebra of $K(O)$.

Proof. The proof has mostly been given above. To complete the proof of (i) one notes that injectivity follows since if $X \in K(M)$ vanishes on the open subset $O$ then $X \equiv 0$. In (ii) the last sentence is clear and the proof is completed by the following examples. First let $M_{1}=\mathbb{R}^{2}$ with positive definite metric $g_{1}$ given by $e^{x^{2}+y^{2}}\left(d x^{2}+d y^{2}\right)$ so that $K\left(M_{1}\right)$ is 1-dimensional and spanned by the Killing vector field $x \frac{\partial}{\partial y}-y \frac{\partial}{\partial x}$ which has a single zero at the origin. Next let $M_{2}=$ $(-1, \infty) \times \mathbb{R}$ with Lorentz metric $g_{2}$ given in coordinates $t, z$ by $-d t^{2}+2 d t d z+t d z^{2}$ so that $K\left(M_{2}\right)$ is 1-dimensional and spanned by the nowhere-zero Killing vector field $\frac{\partial}{\partial z}$. Note that, in this case, the Killing orbits are 1-dimensional and are timelike for $-1<t<0$, null for $t=0$ and spacelike for $t>0$. Finally let $M_{3}=\mathbb{R}^{2}$ with the usual 2-dimensional Minkowski metric $g_{3}$ so that $K\left(M_{3}\right)$ is 3dimensional. Now let $\left(M^{\prime}, g^{\prime}\right)$ be the metric product of $\left(M_{1}, g_{1}\right)$ and $\left(M_{2}, g_{2}\right)$ so that $M^{\prime}=M_{1} \times M_{2}$ and $g^{\prime}=g_{1} \otimes g_{2}$. Then $K\left(M^{\prime}\right)$ can be checked to be the vector space sum $K\left(M_{1}\right) \oplus K\left(M_{2}\right)$ and is thus 2-dimensional. In $M^{\prime}$, the submanifolds of the form $N^{\prime}=\{(0,0)\} \times N$, where $N$ is any Killing orbit in $M_{2}$, are each 1-dimensional Killing orbits in $M^{\prime}$ which are spacelike (respectively, timelike or null) if the orbit $N$ is spacelike (respectively, timelike or null). From the choice of $\left(M_{2}, g_{2}\right)$, each can occur. All other orbits in $M^{\prime}$ are 2-dimensional and may be spacelike, timelike or null. Again, each possibility can occur. Restricting to the situation when the 1-dimensional orbits $N^{\prime}$ are non-null one sees that the associated 
map $k: K\left(M^{\prime}\right) \rightarrow K\left(N^{\prime}\right)$ is not injective. Next let $(\widetilde{M}, \widetilde{g})$ be the metric product of $\left(M_{1}, g_{1}\right)$ and $\left(M_{3}, g_{3}\right)$. Again $K(\widetilde{M})=K\left(M_{1}\right) \oplus K\left(M_{3}\right)$ and so $\operatorname{dim} K(\widetilde{M})=4$ and $\widetilde{N} \equiv\{(0,0)\} \times M_{3}$ is a 2-dimensional timelike orbit with all other orbits timelike and 3-dimensional. Clearly $\widetilde{N}$ is flat and $\operatorname{dim} K(\widetilde{N})=3$ and so the map $k: K(\widetilde{M}) \rightarrow K(\widetilde{N})$ is not injective. An obvious variant of this argument using the metric product of Euclidean space $\mathbb{R}^{2}$ with the manifold $\mathbb{R}^{2}$ with Lorentz metric $e^{-t^{2}+z^{2}}\left(-d t^{2}+d z^{2}\right)$ and with single independent Killing vector field $z \frac{\partial}{\partial t}+t \frac{\partial}{\partial z}$ yields an example of a space-time $\bar{M}$ with $\operatorname{dim} K(\bar{M})=4$ and which admits a single 2dimensional flat spacelike orbit and with all other orbits 3-dimensional and either timelike $\left(z^{2}>t^{2}\right)$, spacelike $\left(z^{2}<t^{2}\right)$ or null $(t= \pm z)$.

The reason that $k$ may fail to be injective is because a set of Killing vector fields on $M$ may be independent as members of the vector space $K(M)$ but restrict to a dependent set of members of $K(O)$ for some orbit $O$. This is what happens in the examples above where non-trivial members of $K(M)$ actually vanish on $O$. This will be explored further in theorem 7 .

Now define a subset $I_{m} K(M)$ (or, simply, $I_{m}$ if $K(M)$ is clear) of $K(M)$ for $m \in M$ by

$$
I_{m} K(M)\left(=I_{m}\right)=\{X \in K(M): X(m)=0\}
$$

It is clear that $I_{m}$ is a vector subspace of $K(M)$ and, in fact, a subalgebra (the isotropy subalgebra) of $K(M)$ since $X(m)=0$ and $Y(m)=0 \Rightarrow[X, Y](m)=$ 0 . Also, if $X \in K(M)$ and $X(m)=0$, then, by the above definition of $K(M)$, each associated map $\phi_{t}$ of $X$ satisfies $\phi_{t}(m)=m$ and also the derived linear map $\phi_{t *}: T_{m} M \rightarrow T_{m} M$ is a member of the Lorentz group with respect to $g(m)$. If one chooses a coordinate neighbourhood $U$ of $p$ with coordinates $x^{a}$, and hence a basis $\left(\frac{\partial}{\partial x^{a}}\right)_{m}$ for $T_{m} M$, a basis for the Lie algebra of this Lorentz group in this basis is the 6-dimensional Lie algebra (under matrix commutation) of matrices $A_{b}^{a}$ satisfying $g_{a c} A_{b}^{c}+g_{b c} A_{a}^{c}=0$ where $g_{a b}$ are the components of $g(m)$ in these coordinates. Thus the map $f$ which associates $X \in I_{m}$ with its Killing bivector $F_{b}^{a}(m)\left(=X_{, b}^{a}(m)\right.$-see (15)) can be regarded as a (linear) map from $I_{m}$ to the Lie algebra of the Lorentz group. Further, if $Y$ is also in $I_{m}$ with Killing bivector $G$, $f$ maps $Y \rightarrow G^{a}{ }_{b}(m)$ and then $f$ maps $[X, Y]$ to $\left(F^{a}{ }_{c} G^{c}{ }_{b}-G^{a}{ }_{c} F^{c}{ }_{b}\right)(m)$. Thus $f$ is a Lie algebra homomorphism from $I_{m}$ to the Lie algebra of the Lorentz group and, since $X(m)=0, F_{b}^{a}(m)=0 \Rightarrow X \equiv 0$ on $M, f$ is an isomorphism onto its range. Thus $I_{m}$ is isomorphic to a subalgebra of the Lie algebra of the Lorentz group. Also, for any $m \in M$, the map $g: K(M) \rightarrow T_{m} M$ given by $X \rightarrow X(m)$ is linear with range $\Delta(m)(\operatorname{see}(1))$ and kernel $I_{m}$. Since $\operatorname{dim} \Delta(m)=\operatorname{dim} O$, where $O$ is the orbit through $m$, it follows that $\operatorname{dim} K(M)=\operatorname{dim} O+\operatorname{dim} I_{m}$.

If $m \in M$ is a zero of $X \in K(M)$ then a certain amount of information is available about the Ricci and Weyl tensors at $m$. This information depends on the subgroup $I_{m}$ of the Lorentz group, that is, on the Killing bivectors of those members of $K(M)$ which vanish at $\mathrm{m}$. The following theorem summarises the situation, the proof of which can be found in [12]-14. It is remarked here that if $0 \not \equiv X \in K(M)$ with $X(m)=0(m \in M)$ then the relationship $£_{X} R_{a b}=0$ evaluated at $m$ using (10) gives $F_{a}{ }^{c} R_{c b}+F_{b}{ }^{c} R_{c a}=0$. Thus the 2-space of $T_{m} M$ representing the blade of $F$ (if $F$ is simple at $m$ ) or the pair of 2-spaces of $T_{m} M$ representing the canonical pair of blades of $F$ (if $F$ is not simple at $m$ ) are each eigenspaces of the Ricci tensor and hence the latter is, in this sense, degenerate [15].

Theorem 4. Let $M$ be a space-time, let $m \in M$ and suppose that $I_{m}$ is not trivial. Then the Petrov type at $m$ is $O, N$ or $D$ and the Ricci tensor admits at least one 
eigenvalue degeneracy. In detail, and identifying members of $I_{m}$ with their bivectors at $m$,

(i) if any of these (simple or non-simple) bivectors is non-null the Petrov type at $m$ is $O$ or $D$ whilst if any is null the Petrov type at $m$ is $O$ or $N$ (and in either case the Segre type of the Ricci tensor is degenerate),

(ii) if $\operatorname{dim} I_{m}=3$ the Weyl tensor vanishes at $m$ and the Ricci tensor has Segre type either $\{1,(111)\},\{(1,11) 1\}$ or $\{(211)\}$ (or the degeneracy of each of the first two of these types),

(iii) if $\operatorname{dim} I_{m} \geq 4$ then at $m$ the Weyl tensor is zero and the Ricci tensor satisfies $R_{a b}=R / 4 g_{a b}$.

\section{The Orbit Structure of $K(M)$}

Here the relationship between $K(M)$ and the possible dimensions of the associated orbits will be investigated. To do this a distinction must be made between those orbits which are 'stable' with respect to their nature or dimension (or both) and those which are not. Let $O$ be a proper orbit. Then either $O \subseteq S_{p}, O \subseteq T_{p}$ or $O \subseteq N_{p}$ for some $p, 1 \leq p \leq 3$. Call $O$ stable if $O$ is entirely contained in either $\operatorname{int} S_{p}$, int $T_{p}$ or int $N_{p}$ or, equivalently, if for each $m \in O$ there exists a neighbourhood $U$ of $m$ such that all orbits through all points of $U$ are of the same nature and dimension as $O$. Thus if $O$ is stable then nearby orbits have the same nature (spacelike, timelike or null) and dimension as $O$. Next call $O$ dimensionally stable if $O$ is entirely contained in some int $V_{p}(1 \leq p \leq 3)$ or, equivalently, if for $m \in O$ there is an open neighbourhood $U$ of $O$ such that all orbits through all points of $U$ are of the same dimension as $O$. Clearly if $O$ is stable it is dimensionally stable. Thus, for example, in the extended Schwarzschild solution one has 3-dimensional Killing orbits everywhere which are timelike and stable outside the Schwarzschild radius, spacelike and stable inside this radius and null and unstable (but dimensionally stable) on it. For the generic F.R.W. metrics the orbits are everywhere 3-dimensional spacelike and stable whilst for the usual Einstein static universe there is a single 4-dimensional orbit. Of the examples described in the proof of theorem 3, the space-time $M^{\prime}$ has the submanifolds $N^{\prime}$ as 1-dimensional dimensionally unstable (and hence unstable) orbits whilst all others are 2-dimensional and each is stable except the null one (which is, however, dimensionally stable). The space-time $\widetilde{M}$ has $\widetilde{N}$ as an unstable (and dimensionally unstable) orbit with all other stable and the space-time $\bar{M}$ admits a 2-dimensional spacelike unstable (and dimensionally unstable) orbit with all others 3-dimensional and each of these stable except the null one (which is, however, dimensionally stable).

Theorem 5. Let $M$ be a space-time.

(i) If $O$ is an orbit of $K(M)$ such that $O \cap \operatorname{int} S_{p} \neq \emptyset$ (respectively, $O \cap \operatorname{int} T_{p} \neq$ $\emptyset$ or $\left.O \cap \operatorname{int} N_{p} \neq \emptyset\right)$ for some $p(1 \leq p \leq 3)$, then $O \subseteq \operatorname{int} S_{p}$ (respectively, $O \subseteq \operatorname{int} T_{p}, O \subseteq \operatorname{int} N_{p}$ ) and is hence stable. Thus the open dense subset $M \backslash Z$ of $M$ in the decomposition ( 7 ) is the union of all stable orbits of $M$ whilst the nowhere dense closed subset $Z$ is the union of all the unstable orbits of $M$. Similarly if $O \cap \operatorname{int} V_{p} \neq \emptyset(1 \leq p \leq 3)$ then $O \subseteq$ int $V_{p}$ and $O$ is dimensionally stable. Thus the open dense subset $M \backslash Z_{1}$ of $M$ in the decomposition (6) is the union of all dimensionally stable orbits of $M$ whilst the nowhere dense closed subset $Z_{1}$ is the union of all dimensionally unstable orbits.

(ii) In the decomposition (7) of $M, S_{3}$ and $T_{3}$ are open subsets of $M$. Thus each 3-dimensional spacelike or timelike orbit of $K(M)$ is stable. 
Proof. (i) Let $O$ be such an orbit with $O \cap \operatorname{int} S_{p} \neq \emptyset$ and let $m \in O \cap \operatorname{int} S_{p}$. If $m^{\prime}$ is any other point of $O$ there exists an open neighbourhood $U$ of $m$ and a local isometry $f$ of the form (2) which is defined on $U$ such that $f(m)=m^{\prime}$ and $U \subseteq$ int $S_{p}$. The orbits intersecting $U$ are $p$-dimensional and spacelike and similarly for each point in the open neighbourhood $f(U)$ of $m^{\prime}$. Thus $O \subseteq \operatorname{int} S_{p}$ and a similar argument suffices for the timelike and null cases. The rest of the proof is similar.

(ii) Let $O$ be a 3-dimensional spacelike orbit. If $m \in O$ construct a Gauss coordinate domain $U$ about $m$ such that each point of $U$ lies on a time-like geodesic which intersects $O$ orthogonally. Denoting the tangent vector of such a geodesic by $K(t)$, with $t$ an affine parameter, one has the well-known result (see e.g. 16]) that if $X \in K(M)$ then $X^{a} k_{a}$ is constant along the geodesic (and hence zero along the geodesic since it vanishes on $O$ ). Thus at any point of $U$ the orbit of $K(M)$ is orthogonal to a timelike vector and is hence spacelike with dimension at most three. However, the rank theorem ensures that $U$ can be reduced (if necessary) so that the orbit through any point of $U$ is of dimension at least three. Thus each orbit through $U$ is 3-dimensional and spacelike and so $U \subseteq \operatorname{int} S_{3}$ and $O$ is stable. A similar argument applies in the timelike case and so $S_{3}=\operatorname{int} S_{3}$ and $T_{3}=\operatorname{int} T_{3}$ in $(7)$.

It is remarked that, as a consequence of theorem 5(i), there is an open neighbourhood of any point in the open dense subset $M \backslash Z$ (i.e. of "almost any" point of $M$ ) in which the orbits are of the same dimension and nature.

It was mentioned at the beginning of section 4 that $\operatorname{dim} K(M) \leq 10$. If $\operatorname{dim} K(M)$ $=10$ then $M$ is of constant curvature and if $M$ is of constant curvature then each $m \in M$ admits a connected neighbourhood $U$ such that, with its induced metric, $U$ satisfies $\operatorname{dim} K(U)=10$. If $\operatorname{dim} K(M)=9$ then, as will be seen later (theorem 6 ), the only possible orbits are 4-dimensional and so $M=V_{4}$ from theorem 2 . But then a result at the end of section 4 shows that $\operatorname{dim} I_{m}=9-4=5$ for each $m \in M$ and this is a contradiction since the Lorentz group has no 5 -dimensional subgroups. Thus $\operatorname{dim} K(M)=9$ is impossible. If $\operatorname{dim} K(M)=8$ then, again, theorem 6 will show that $M=V_{4}$ and hence that $\operatorname{dim} I_{m}=4$ at each $m \in M$. It follows from theorem 4 that $M$ is of constant curvature and so, locally, admits a 10-dimensional Killing algebra. Thus, if $m \in M$, there exists an open neighbourhood $U$ of $m$ such that $\operatorname{dim} K(U)=10$. (c.f Yegerov's theorem quoted in [17]). It should be remarked at this point that the impossibility of $\operatorname{dim} K(M)=9$ may be thought an immediate consequence of Fubini's theorem [18] and which appears to rule out $\operatorname{dim} K(M)=1 / 2 n(n+1)-1$ for a manifold $M$ of dimension $n$ and with metric $g$. As far as the present author can tell, Fubini's theorem only deals with the positive definite case but, fortunately, can be extended to cover (almost) all signatures and, in particular, applies to space-times (see appendix).

Theorem 6. Let $M$ be a space-time. Then the following hold for the orbits of $K(M)$.

(i) If there exists a 3-dimensional null orbit, then $3 \leq \operatorname{dim} K(M) \leq 7$. If, however, there exists a null dimensionally stable 3-dimensional orbit or any non-null 3-dimensional orbit then $3 \leq \operatorname{dim} K(M) \leq 6$.

(ii) If there exists a 2-dimensional null orbit, then $2 \leq \operatorname{dim} K(M) \leq 5$ and if there exists a 2-dimensional non-null orbit, $2 \leq \operatorname{dim} K(M) \leq 4$. If there exists any 2-dimensional dimensionally stable orbit, $2 \leq \operatorname{dim} K(M) \leq 3$.

(iii) If there exists a 1-dimensional null orbit, $1 \leq \operatorname{dim} K(M) \leq 5$ and if there exists a 1-dimensional non-null orbit, $1 \leq \operatorname{dim} K(M) \leq 4$. If there exists a 1-dimensional dimensionally stable orbit, $\operatorname{dim} K(M)=1$. 
Proof. (i) If any 3-dimensional orbit $O$ exists then $\operatorname{dim} K(M) \geq 3$. Suppose, in addition, that $O$ is null (and not necessarily dimensionally stable). Let $m \in O$ and choose independent members $X^{\prime}, Y^{\prime}$ and $Z^{\prime}$ in $K(M)$ which span $O$ at $m$. Then in some coordinate neighbourhood $U$ of $m$ the smooth vector field $k$ in $U$ with components $k^{a}=\varepsilon^{a}{ }_{b c d} X^{\prime b} Y^{\prime c} Z^{\prime d}$, where $\varepsilon$ denotes the usual alternating symbol, is orthogonal to $X^{\prime}, Y^{\prime}$ and $Z^{\prime}$ on $U$. Also, since $X^{\prime}, Y^{\prime}$ and $Z^{\prime}$ span $O$ at $m, U$ may (and will) be chosen so that none of $X^{\prime}, Y^{\prime}, Z^{\prime}$ and $k$, can vanish at any point of $U$. Thus, since $O$ is null, $k$ is null and tangent to $O$ on $O^{\prime} \equiv U \cap O$. Now let $X$ be a non-trivial member of $K(M)$. Then $X$ is tangent to $O$ and hence orthogonal to $k$ on $O^{\prime}$. Thus $\left(X^{a} k_{a}\right)_{; b} p^{b}=0$ on $O^{\prime}$ where $p^{a}$ are the components of any tangent vector to any curve in $M$ lying in $O^{\prime}$. Suppose $X(m)=0$. Then evaluating this differential condition at $m$, using (10), shows that the Killing bivector $F$ of $X$ satisfies $F_{a b} p^{a} k^{b}=0$ at $m$ for any $p \in T_{m} M$ tangent to $O$. It follows from elementary bivector algebra that $F_{a b} k^{b}=\lambda k_{a}$ at $m(\lambda \in \mathbb{R})$ and that at most four independent bivectors at $m$ can have this property (since $k$ is null). Thus $\operatorname{dim} I_{m} \leq 4$ and so $\operatorname{dim} K(M) \leq 7$.

Now suppose the orbit $O$ is 3-dimensional and either null and dimensionally stable or non-null (and hence necessarily stable by theorem 5). Let $m \in O$ and $U$ an open neighbourhood of $m$ such that each orbit intersecting $U$ has the same dimension and type as $O$. By the same argument as in the previous paragraph, one can (reducing $U$ if necessary) choose a smooth vector field $k$ on $U$ everywhere orthogonal to the orbits in $U$. Thus if $X$ is a non-trivial member of $K(M), X^{a} k_{a}=$ 0 on $U$. Now suppose $X(m)=0$ and evaluate the condition $\left(X^{a} k_{a}\right)_{; b}=0$ at $m$ to see that the Killing bivector $F$ of $X$ satisfies $F_{a b} k^{b}=0$ at $m$. Since only three independent bivectors $F$ may have this property one sees that $\operatorname{dim} I_{m} \leq 3$ and hence that $\operatorname{dim} K(M) \leq 6$. [In fact if $O$ is non-null, the map $K(M) \rightarrow K(O)$ given by $X \rightarrow \tilde{X}$ is injective (theorem 3 ) and the above result follows in this case ( since $\operatorname{dim} K(O) \leq 6)$ without appeal to the dimensional stability of $O$.]

(ii) If there exists a 2-dimensional orbit $O$ then $\operatorname{dim} K(M) \geq 2$. Let $m \in O$ and let $Y, Z \in K(M)$ such that $Y$ and $Z$ span $O$ at $m$. Now let $U$ be a coordinate neighbourhood of $m$ such that $G_{a b} \equiv 2 Y_{[a} Z_{b]}$ is a smooth simple nowhere-zero bivector on $U$ and (hence so is its dual $G_{a b}^{*}$ ). It follows (see, e.g. [19]) that, reducing $U$ if necessary, one may write $G_{a b}^{*}=2 P_{[a} Q_{b]}$ for some smooth vector fields $P$ and $Q$ on $U$ and then $P$ and $Q$ span the orthogonal complement of the tangent space to $O$ at each point of $O \cap U$. Now suppose $\operatorname{dim} K(M)>2(=\operatorname{dim} O)$. Then, as in the proof of part (i), there exists $X \in K(M), X \not \equiv 0$, such that $X(m)=0$. Also, since $X$ is tangent to $O, X^{a} P_{a}=X^{a} Q_{a}=0$ on $O$. Thus

$$
\left(X^{a} P_{a}\right)_{; b} Y^{b}=\left(X^{a} P_{a}\right)_{; b} Z^{b}=\left(X^{a} Q_{a}\right)_{; b} Y^{b}=\left(X^{a} Q_{a}\right)_{; b} Z^{b}=0
$$

On evaluating (18) at $m$ one finds that the bivector $F$ of $X$ satisfies, at $m$, the conditions

$$
F_{a b} P^{a} Y^{b}=F_{a b} P^{a} Z^{b}=F_{a b} Q^{a} Y^{b}=F_{a b} Q^{a} Z^{b}=0
$$

If $O$ is non-null, (19) represents four independent conditions on $F$ at $m$ and thus there are only two independent bivectors in the vector space of bivectors at $m$ satisfying them. In this case (c.f part (i)) one finds $2 \leq \operatorname{dim} K(M) \leq 4$. If $O$ is null, (19) represent only three restrictions (since in this case the tangent space to $O$ is a null 2-space and intersects its orthogonal complement in a null direction and so $X, Y, P$ and $Q$ do not yield independent tangent vectors at any point of $O$ ). Thus in this case there are exactly three independent bivectors satisfying (19) and so $2 \leq \operatorname{dim} K(M) \leq 5$. If $O$ is a dimensionally stable orbit, then by following closely the proof of part (i) one has for $m \in O, X \in K(M), X \not \equiv 0, X(m)=0$, the 
consequences $X^{a} P_{a}=X^{a} Q_{a}=0$ on some open neighbourhood of $m$ and so

$$
\left(X^{a} P_{a}\right)_{; b}=\left(X^{a} Q_{a}\right)_{; b}=0\left(\Rightarrow F_{a b} P^{b}=F_{a b} Q^{b}=0 \text { at } m\right)
$$

There is only one independent bivector satisfying the bracketed consequence in (20) and so $\operatorname{dim} I_{m} \leq 1$. Hence $2 \leq \operatorname{dim} K(M) \leq 3$.

(iii) The proof of (iii) is similar. If $O$ is a 1-dimensional orbit and if $m \in O$ choose $X \in K(M)$ which spans $O$ at $m$ and a coordinate neighbourhood $U$ of $m$ in which $X=\partial / \partial x^{1}$. Then the three independent vector fields on $U$ whose associated covector fields on $U$ are $d x^{a}(a=2,3,4)$ are orthogonal to $X$ on $U$. The result now follows with the last part of it being simply the statement that if $X, Y \in K(M)$ and $X=\phi Y$ over some non-empty open subset $U$ of $M$ for some function $\phi: U \rightarrow \mathbb{R}$, then $\phi$ is constant and $X=\phi Y$ on $M$ [16].

Regarding theorem 6(i) the author does not know of an example of a space-time $M$ with $\operatorname{dim} K(M)=7$ and where a dimensionally unstable 3-dimensional null orbit (or orbits) exist. If such an $M$ exists then theorem 6 shows that all other orbits are 4-dimensional and clearly $V_{0}=\emptyset$ in the decomposition (7) otherwise for $m \in V_{0}$ one would obtain the contradiction $\operatorname{dim} I_{m}=7$. Thus, in (7), one would have $M=V_{4} \cup Z$ where $Z=N_{3}$ (and int $Z=\emptyset$ ).

Also regarding theorem $6(\mathrm{i})$ the situation with $\operatorname{dim} K(M)=5$ and $O$ a 3dimensional spacelike orbit is impossible since, for $m \in O$, one would have $I_{m}$ isomorphic to a 2-dimensional subalgebra of $s o(3)$, which does not exist. The nonhomogeneous plane waves show, however, that $\operatorname{dim} K(M)=5$ (or 6) and $O$ a 3 -dimensional null (and stable) orbit is possible. Now consider the situation when $\operatorname{dim} K(M)=5$ and $O$ is a 3 -dimensional timelike (necessarily stable) orbit. It is sometimes argued that this implies that $\operatorname{dim} K(O)=5$ and that this is impossible by appealing to Fubini's theorem. However, theorem 3 shows that $\operatorname{dim} O \geq 5$ and it can then be established that $O$ is a 3 -dimensional manifold of constant curvature and so $O$ admits a local 6-dimensional Killing algebra about each of its points. It is not immediately obvious that this contradicts the fact that $\operatorname{dim} K(M)=5$. Also, the above method of contradicting the situation when $O$ is spacelike fails here since $s o(1,2)$ admits a 2-dimensional subalgebra. To proceed further consider the open submanifold $T_{3}$ of $M$ where the orbits are timelike and 3-dimensional and let $m \in T_{3}$. Then $I_{m}$ is a 2-dimensional subalgebra of $o(1,2)$ generating the subgroup of null rotations of the 3 -dimensional Lorentz group. This is easily checked either by direct computation or by noting that it must generate a subgroup of the 4 dimensional Lorentz group which fixes a spacelike vector (the normal to the orbit) and then checking the possibilities from this latter Lorentz group (see, e.g [20, 21]). Next, using an argument similar to that in the proof of theorem 6 , one can construct a coordinate neighbourhood $U \subseteq T_{3}$ of $m$ and a smooth geodesic vector field $Y$ on $U$ which is everywhere orthogonal to the orbits and which gives Gauss coordinates on $U$ in which the metric is

$$
d s^{2}=d y^{2}+g_{\alpha \beta} d x^{\alpha} d x^{\beta}
$$

$(\alpha, \beta=0,1,2)$ and where $Y^{a}=\delta_{3}^{a}, Y_{a}=y_{, a}=\delta_{a}^{3}, Y^{a} Y_{a}=1$ and $Y_{a ; b} Y^{b}=0$. At each point $m^{\prime}$ of $U$ one may extend $Y\left(m^{\prime}\right)$ to a null tetrad $l, n, x, Y\left(m^{\prime}\right)$ and then $I_{m}$ may be regarded as being spanned by the bivectors $l_{[a} n_{b]}$ and $l_{[a} x_{b]}$. Theorem 4 and the remarks preceding it can then be used to show that $T_{3}$ is a conformally flat region and that, in $U$, the Ricci tensor takes the form $R_{a b}=\alpha(y) g_{a b}+\beta(y) Y_{a} Y_{b}$ (where, since each subset of $U$ of constant $y$ lies in an orbit, the Ricci eigenvalues $\alpha$ and $\beta$ depend, at most, on $y$ ). The conformally flat Bianchi identity is

$$
R_{c[a ; b]}=\frac{1}{6} g_{c[a} R_{, b]}
$$


where $R \equiv R_{a b} g^{a b}$ is the Ricci scalar and a contraction of (22) with $Y^{a}$ yields

$$
6 \beta Y_{a ; b}=(2 \dot{\alpha}-\dot{\beta})\left(g_{a b}-Y_{a} Y_{b}\right)(. \equiv d / d y)
$$

In the open subregion $A \subseteq T_{3}$ on which $\beta \neq 0$ one may solve (23) for $Y_{a ; b}$ and by a standard argument [use (21) and (23) to get $Y_{a ; b}=-\Gamma_{a b}^{3} \Rightarrow\left(\frac{2 \dot{\alpha}-\dot{\beta}}{6 \beta}\right) g_{\alpha \beta}=$ $1 / 2 g_{\alpha \beta, 3} \Rightarrow g_{\alpha \beta}=h(y) q_{\alpha \beta}$ for a positive function $h(y)$ and functions $q_{\alpha \beta}$ independent of $y$ ] cast the metric locally into the form

$$
d s^{2}=d y^{2}+S^{2}(y) q_{\alpha \beta} d x^{\alpha} d x^{\beta}
$$

for a smooth function $S$ and where $q_{\alpha \beta}$ are the components of a (3-dimensional constant curvature) Lorentz metric in the orbit through $m$. The metric (24) is just the spacelike equivalent of a F.R.W. metric and it easily follows that $T_{3}$ admits, locally, a Lie algebra of Killing vector fields of dimension at least six. If $B$ is the subregion of $T_{3}$ on which $\beta=0$ then (23) shows that $\alpha$ is constant on each component of int $B$ and so int $B$ is an open subregion of $M$ on which the Einstein space and conformally flat conditions hold and so is of constant curvature (and so the local Killing algebra is 10-dimensional). Thus, for $\operatorname{dim} K(M)=5$, each point of the open dense subset $A \cup \operatorname{int} B$ of $T_{3}$ admits, locally, a Killing algebra of dimension greater than five. Thus from the physical viewpoint, if one wishes to study symmetry corresponding to $\operatorname{dim} K(M)$ being exactly five (even locally) then on $T_{3}$ (and on $M$ if all orbits are 3-dimensional and timelike) such conditions cannot be achieved.

All the other dimensionally stable possibilities in theorem 6 can be constructed [14. The examples given after theorem 3 confirm the existence of space-times with $\operatorname{dim} K(M)=4$ and $\operatorname{dim} O=2$ with $O$ not dimensionally stable and either timelike or spacelike, and with $\operatorname{dim} K(M)=2$ and $\operatorname{dim} O=1$ with $O$ not dimensionally stable and either timelike, spacelike or null. Now consider the space-time metrics

$$
\begin{gathered}
d s^{2}=-d t^{2}+e^{x^{2}+y^{2}+z^{2}}\left(d x^{2}+d y^{2}+d z^{2}\right) \\
d s^{2}=d z^{2}+e^{-t^{2}+x^{2}+y^{2}}\left(-d t^{2}+d x^{2}+d y^{2}\right)
\end{gathered}
$$

each on the manifold $R^{4}$. For each of these space-times $\operatorname{dim} K(M)=4$ with $K(M)$ being spanned by the global Killing vector fields with components (in the coordinates $t, x, y, z)$ given by $(1,0,0,0),(0,0, z,-y),(0, y,-x, 0)$ and $(0, z, 0,-x)$ for $(25)$ and by $(0,0,0,1),(x, t, 0,0),(y, 0, t, 0)$ and $(0,-y, x, 0)$ for $(26)$. Then $(25)$ admits the submanifold $x=y=z=0$ as a 1-dimensional timelike orbit which is not dimensionally stable, with all other orbits stable, 3-dimensional and timelike. Similarly, (26) admits the submanifold $t=x=y=0$ as a 1-dimensional spacelike orbit which is not dimensionally stable, with all other orbits stable, 3-dimensional and timelike. Thus many of the dimensionally unstable possibilities in theorem 6 parts (ii) and (iii) can occur. There are, however, some that cannot. For example, if $\operatorname{dim} K(M)=3$ and $O$ is a 1-dimensional timelike orbit then, for $m \in O$, one again has the contradiction that $I_{m}$ is a 2-dimensional subalgebra of $s o(3)$. The other situations allowed by this theorem are, at least to the author, as yet unresolved.

\section{Further Remarks}

For space-times admitting Killing symmetry and for which a non-trivial isotropy $I_{m}$ exits, much valuable information is available from the bivectors at $m$ associated with the members of $I_{m}$. At this point it is useful to note (by adopting the discussion of section 4) that on a 2-dimensional manifold a non-zero bivector is necessarily a non-singular matrix and so a zero of a non-trivial Killing vector field on such a manifold is necessarily isolated. Similarly, on a 3-dimensional manifold, a non-zero 
bivector has rank equal to two and is hence a singular matrix. Thus a zero of a non-trivial Killing vector field in this case is never isolated but, rather (should a zero exist), its zeros constitute (locally) a 1-dimensional submanifold.

From the calculations in the proof of theorem 6, and given $m \in M$ and the nature (spacelike, timelike or null) and dimension of the orbit $O$ through $p$ (assumed proper) and whether $O$ is dimensionally stable or not, it is straightforward to write down a basis for the vector space of Killing bivectors at $p$ for members of $I_{m}$. (It is not claimed that all possibilities not so far eliminated from theorem 6 can exist.) From this information (or by an easy direct calculation) it turns out that whatever the nature of $O$, provided it is dimensionally stable, any Killing bivector at $m$ arising from a non-trivial member $X$ of $I_{m}$ is simple and its blade is tangent to $O$ at $m$ (and note that if $I_{m}$ is not trivial then $O$ cannot be 1-dimensional). Thus $m$ is not isolated.

For an orbit $O$ which is not dimensionally stable the calculations for theorem 6 again show that the Killing bivector at $m \in O$ arising from some $X \in I_{m}$ is also simple (and so $m$ is not isolated) except, possibly, in the cases when $O$ is either 1-dimensional and null, 2-dimensional and non-null or 3-dimensional and null. For example, consider the space-time $\widetilde{M}=M_{1} \times M_{3}$ constructed during the proof of theorem 3 and let $X$ be non-trivial member of $K\left(M_{1}\right)$ which vanishes at the origin $o$ of $M_{1}$. Now let $Y$ be the vector field on $M_{3}$ given in the obvious coordinates by $Y=z \partial / \partial t+t \partial / \partial z$. Then $Y$ vanishes at the origin $o^{\prime}$ of $M_{3}$ and the vector field sum $X \oplus Y$ is a member of $K\left(\widetilde{M}\right.$ which vanishes at $\left(o, o^{\prime}\right) \in \widetilde{M}$ and its Killing bivector there is easily checked to be non-simple. In this case $\operatorname{dim} K(\widetilde{M})=4$ and the orbit through $\left(o, o^{\prime}\right)$ is 2-dimensional, timelike and not dimensionally stable. Finally, consider the example of the space-time $M=R^{4}$ with metric

$$
d s^{2}=e^{-t^{2}+x^{2}}\left(-d t^{2}+d x^{2}\right)+e^{y^{2}+z^{2}}\left(d y^{2}+d z^{2}\right)
$$

Here a basis for $K(M)$ can be taken as consisting of the vector fields $X=t \partial / \partial x+$ $x \partial / \partial t$ and $Y=y \partial / \partial z-z \partial / \partial y$ and so $\operatorname{dim} K(M)=2$. The Killing vector field $X+Y$ vanishes at the origin and its bivector there is non-simple. The origin is itself a (non-proper) orbit and the isotropy subalgebra there is 2-dimensional. This example also possesses 1-dimensional spacelike, timelike and null orbits which are not dimensionally stable in the submanifolds $\left\{(t, x, 0,0) t, x \in R, t^{2}+x^{2} \neq 0\right\}$ and similar spacelike ones in the submanifolds $\left\{(0,0, y, z) y, z \in R, y^{2}+z^{2} \neq 0\right\}$. All other orbits are 2-dimensional, stable and can be spacelike, timelike or null.

It was mentioned, following the proof of theorem 3, that an independent set of Killing vector fields on $M$ when restricted to an orbit $O$ of $K(M)$ may no longer be independent. However, in all the examples given of this phenomenon, $O$ was not dimensionally stable or 3 -dimensional. This feature turns out to be general as the next theorem shows.

Theorem 7. Let $M$ be a space-time and let $X^{1}, \ldots, X^{n}$ be independent members of $K(M)$. If an orbit $O$ is 3-dimensional or is any dimensionally stable orbit of $K(M)$ then the restrictions $\widetilde{X^{1}}, \ldots, \widetilde{X^{n}}$ to $O$ are independent vector fields on $O$ (and hence independent members of $K(O)$ if $O$ is non-null). Said another way, a nontrivial Killing vector field cannot vanish on such an orbit $O$ (that is, the Lie algebra homomorphism $X \rightarrow \widetilde{X}$ is injective).

Proof. Let $X$ be a non-trivial member of $K(M)$ and let $O$ be an orbit of $K(M)$ such that $X$ vanishes on $O$. It follows immediately from theorem 3(ii) that $O$ cannot be 3-dimensional and from theorem 6(iii) that $O$ cannot be dimensionally stable and 1-dimensional. So let $O$ be dimensionally stable with $\operatorname{dim} O=2$ and, following the proof of theorem 6 since $O$ is dimensionally stable, for $m \in O$ let 
$U$ be a coordinate neighbourhood of $m$ in $M$ such that there exist $Y, Z \in K(M)$ with $Y\left(m^{\prime}\right)$ and $Z\left(m^{\prime}\right)$ spanning the tangent space to $O$ at each $m^{\prime} \in U$ and such that there exist smooth vector fields $W$ and $T$ on $U$ which are orthogonal to the (2-dimensional) orbits of $K(M)$ at each point of $U$. Then $X^{a} W_{a}=X^{a} T_{a}=0$ on $U$ and so differentiating these equations and evaluating on $O$ (where $X$ vanishes) gives $F_{a b} W^{b}=F_{a b} T^{b}=0$ at each point of $O$, where $F$ is the Killing bivector of $X$. But since $X$ vanishes on $O$ it also follows that, on $O, X^{a}{ }_{; b} Y^{b}=X^{a}{ }_{b b} Z^{b}=0$ and so, on $O, F_{a b} Y^{b}=F_{a b} Z^{b}=0$. It is now clear that, whether $O$ is null or non-null, $F$ (and $X$ ) vanishes on $O$ and hence one has the contradiction that $X \equiv 0$ on $M$. The result follows.

\section{Appendix (Fubini's TheOREM)}

For a manifold $M$ of dimension $n$ and with metric $g$ of arbitrary signature a theorem, often quoted in the literature and due to Fubini [18, says that the Lie algebra $K(M)$ cannot have dimension $\frac{1}{2} n(n+1)-1$ (that is, one less than the maximum for that dimension). However, the proof in [18 (see also [16]) and its reliance on Bianchi's (positive definite) results recently reprinted in 22] suggest that Fubini's theorem should be revisited. It was, of course, shown earlier that Fubini's theorem holds for a space-time $M$, that is, $K(M)$ cannot be 9-dimensional. To see that Fubini's theorem holds for almost all dimensions and signatures consider the orthogonal (Lie) group $S O(p, q)(p+q=n)$ where, because of the symmetry isomorphism $S O(p, q) \approx S O(q, p)$, one need only consider the case $p \leq q$. This group has dimension $\frac{1}{2} n(n-1)$ and acts on $R^{n}$ as a Lie transformation group in the usual way 11. It can be realised as the symmetry group of the metric $\gamma$ with components $\operatorname{diag}(-1, \ldots,-1,1, \ldots 1)$ where there are $p$ minus and $q$ plus signs. If $H$ is a Lie subgroup of $S O(p, q)$ then, by restriction, $H$ also acts on $R^{n}$ as a Lie transformation group. Thus if $0 \neq v \in R^{n}$ and if $H_{v}$ is the Lie subgroup of $H$ which fixes $v$ and $O_{v}$ is the orbit of $v$ under $H$, one has 1 .

$$
\operatorname{dim} H=\operatorname{dim} H_{v}+\operatorname{dim} O_{v}
$$

Now for $n=3$ and for $n \geq 5$ it is known 23 that if $H$ is a proper Lie subgroup of $S O(n), \operatorname{dim} H \leq \frac{1}{2}(n-1)(n-2)$. It is not true for $n=4$ since $S O(4)$ has a 4 (but no higher)-dimensional subgroup. The following lemma is now useful (c.f. 24]).

Lemma 1. Let $H$ be a Lie subgroup of $S O(p, q)$ with $p \leq q, p+q=n \geq 3$. If for each spacelike $v \in R^{n}, H_{v} \approx S O(p, q-1)$ or for each timelike $v \in R^{n}, H_{v} \approx$ $S O(p-1, q)$, then $H \approx S O(p, q)$.

Proof. Let $x_{1}, \ldots, x_{n}$ be an appropriate orthonormal basis for $R^{n}$. If $p=0$ (the positive definite case, so that each $x_{k}$ is spacelike) then each $H_{x_{k}} \approx S O(n-1)$. Thus the skew self-adjoint (with respect to $\gamma$ ) matrices represented in an obvious notation by $x_{i} \wedge x_{j}(i<j, i \neq k \neq j)$ are in the Lie algebra of $H_{x_{k}}$ since if $G$ is any of these matrices, $G x_{k}=0$. Since this is true for each $k$, the Lie algebra of $H$ coincides with that of $S O(n)$ and the result follows. Now suppose that $p \geq 1$ so that one may assume $x_{1}$ timelike and $x_{2}$ and $x_{3}$ spacelike. If $H_{v} \approx S O(p-1, q)$ for each timelike $v$ then, with $v=x_{1}$, the matrices $x_{i} \wedge x_{j}$ for $2 \leq i<j$ are in the Lie algebra of $H_{v}$ and hence of $H$. Now $x^{\prime}=x_{1}+1 / 2 x_{2}$ is timelike and for $i \geq 3$ the matrices $G_{i}=\left(x_{1}+2 x_{2}\right) \wedge x_{i}$ satisfy $G_{i} x^{\prime}=0$ and so are in the Lie algebra of $H$ for each $i \geq 3$. Hence so are the matrices $x_{1} \wedge x_{i}(i \geq 3)$. A similar consideration of the timelike vector $x_{1}+1 / 2 x_{3}$ then shows that $x_{1} \wedge x_{2}$ is in the Lie algebra of $H$ and hence that $H \approx S O(p, q)$. A similar argument reveals the same conclusion if $H_{v} \approx S O(p, q-1)$ for each spacelike $v$. 
Lemma 2. If $H$ is a proper Lie subgroup of any of the Lie groups $S O(p, q), p \leq$ $q, p+q=n, q \geq 3$, then $\operatorname{dim} H<1 / 2 n(n-1)-1$.

Proof. The remarks following (A1) show the result to be true for $S O(n)(n \neq 4)$. That it holds for $S O(4)$ follows by choosing $0 \neq v \in R^{4}$ such that $H_{v}$ is a proper subgroup of $S O(3)$ (from lemma 1) and so $\operatorname{dim} H_{v} \leq 1$. Since $\operatorname{dim} O_{v}=3$, (A1) shows that $\operatorname{dim} H \leq 4$. Now consider $S O(1, n-1)$ for $n \geq 4$ and choose a timelike $v$ in $R^{n}$ for which $H_{v}$ is a proper subgroup of $S O(n-1)$. Equation (A1) and the previous result for $S O(n)$ show that

$$
\operatorname{dim} H<1 / 2(n-1)(n-2)-1+n-1=1 / 2 n(n-1)-1
$$

An induction argument on $p$ completes the proof.

Thus lemma 2 holds for all signatures except $(0,2),(1,1),(1,2)$ and $(2,2)$ and its failure in the first two of these is clear. It also fails for the signature $(1,2)$ because of the existence of a 2-dimensional Lie subgroup of the 3-dimensional Lorentz group. To see why it fails for signature $(2,2)$ let $t_{1}, t_{2}, x_{1}$ and $x_{2}$ be an orthonormal basis for $R^{4}$ with $t_{1}, t_{2}$ timelike and $x_{1}, x_{2}$ spacelike. Then change to a new basis $l, n, x_{1} x_{2}$ with $l \equiv t_{1}+x_{1}$ and $n \equiv t_{2}+x_{2}$ orthogonal null vectors. Next consider the subspace $W$ of the Lie algebra $o(2,2)$ of $S O(2,2)$ spanned by $l \wedge n, l \wedge x_{1}, l \wedge x_{2}, n \wedge x_{1}$ and $n \wedge x_{2}$. It is easily checked that $W$ is a 5 -dimensional subalgebra by either directly computing the appropriate commutators or, more quickly, by noting that if $A, B \in W$ then $[A, B] l$ and $[A, B] n$ are linear combinations of $l$ and $n$ and hence $[A, B]$, when decomposed in the obvious basis has no term in $x_{1} \wedge x_{2}$. The unique connected 5-dimensional Lie subgroup of $S O(2,2)$ associated with $W$ is precisely the subgroup which fixes the totally null 2-dimensional subspace $N$ of $R^{4}$ spanned by $l$ and $n$ (that is each non-zero member of $N$ is null).

The following 'Fubini theorem' can now be proved.

Theorem 8. Let $M$ be a connected smooth paracompact manifold of dimension $n \geq 3$ admitting a smooth metric $g$ of signature $(p, q)$ with $p \leq q$ and $q \geq 3$. Suppose $\operatorname{dim} K(M) \neq 1 / 2 n(n+1)$. Then $\operatorname{dim} K(M)<1 / 2 n(n+1)-1$.

Proof. Let $m \in M$ and $O$ be the orbit associated with $K(M)$ through $m$. Then $O$ is a smooth integral manifold of $K(M)$ and $\operatorname{dim} K(M)=\operatorname{dim} O+\operatorname{dim} K_{m}^{*}$. If $\operatorname{dim} O<n$, arguments such as those used in the proof of theorem 6 show that $K_{m}^{*}$ is restricted and so $\operatorname{dim} K_{m}^{*}<1 / 2 n(n-1)-1$ from lemma 2 . It follows that if $\operatorname{dim} K(M)$ is not the maximum possible then

$$
\operatorname{dim} K(M)<n+1 / 2 n(n-1)-1=1 / 2 n(n+1)-1
$$

The signatures not covered by the theorem are, again $(0,2),(1,1),(1,2)$ and $(2,2)$. In the first two of these the final conclusion of the theorem is false since the ordinary cylinder and the 'Lorentz cylinder' obtained, respectively, by identifying the points $(x, t)$ and $(x+k, t)$ for each $k \in \mathbb{Z}$, in $\mathbb{R}^{2}$, on which the standard Euclidean and Minkowski metrics have been placed, have a 2-dimensional Killing algebra. However, if a 2-dimensional manifold $M$ has $\operatorname{dim} K(M)=2$, it is easily checked from theorem 6 that the orbits are 2-dimensional over an open dense subset $A$ of $M$ and then [16] that $A$ and hence $M$ have constant curvature. Thus the Killing algebra is locally 3 -dimensional. In the $(1,2)$ and $(2,2)$ cases the situation is, to the best of the author's knowledge, unresolved. In the $(1,2)$ case, if $\operatorname{dim} K(M)=5$, one again has constant curvature on $M$ and hence a local 6-dimensional Killing algebra 25. It is easily checked from [26, 27] that in the $(1,1),(0,2)$ and $(1,2)$ signatures, if $M$ is simply connected, $\operatorname{dim} K(M) \neq 1 / 2 n(n+1)-1$.

It is remarked that the result in lemma 2 can be strengthened. 
Lemma 3. Let $H$ be a proper Lie subgroup of $S O(p, q), p \leq q, p+q=n, q \geq 5$. Then $\operatorname{dim} H \leq 1 / 2(n-1)(n-2)+p$. The result also holds for the signatures $(0,2),(0,3),(1,2),(1,3)$ and $(2,2)$.

The proof uses the result for $S O(n)$ quoted earlier and then essentially the argument given in lemma 2 and proceeds by induction from equations like (A1) so that for $v$ timelike $(p \geq 1)$

(A4) $\quad \operatorname{dim} H \leq n-1+1 / 2(n-2)(n-3)+p-1=1 / 2(n-1)(n-2)+p$

This proof and that of lemma 2 are modelled on the work in [24] where lemma 3 for Lorentz signature was established. A further strengthening of lemma 2 and the Fubini theorem can then be achieved. 


\section{REFERENCES}

[1] F. Brickell and R.S. Clark. Differential Manifolds, Van Nostrand, (1970).

[2] P. Stefan. Proc. London Math. Soc. 29, (1974), 699.

[3] S. Kobayashi and K. Nomizu. Foundations of Differential Geometry Vol 1, Interscience, New York, (1963).

[4] R. Abraham, J.E. Marsden and T. Ratiu. Manifolds, Tensor Analysis and Applications. Springer 2nd edition, (1988)

[5] H.J.Sussmann. Trans. Am. Math. Soc. 180, (1973), 171.

[6] R. Hermann. International Symposium on Nonlinear Differential Equations and Nonlinear Mechanics, Academic Press, New York, (1963), 325.

[7] P. Stefan. J. London Math. Soc. 21, (1980), 544.

[8] G.S. Hall. Class. Quant. Grav. 13, (1996), 1479.

[9] G.S. Hall. The Petrov Lectures in the Proceedings of the Volga Summer School XII, University of Kazan, (2000).

[10] W.L. Chow. Math. Ann. 117, (1939), 98.

[11] G.S. Hall. Gen. Rel. Grav. 20, (1988), 671.

[12] G.S. Hall. J. Math. Phys. 31, (1990), 1198.

[13] J. Ehlers and W. Kundt. in Gravitation: An Introduction to Current Research ed. L. Witten. Wiley, New York, (1962).

[14] H. Stephani, D. Kramer, M.A.H. MacCallum, C. Hoenselaers and E. Herlt. Exact Solutions of Einstein's Field Equations, Cambridge University Press, (2003).

[15] G.S. Hall and C.B.G. McIntosh. Int. J. Theor. Phys. 22, (1983), 469.

[16] L.P. Eisenhart. Riemannian Geometry, Princeton University Press, (1966).

[17] A.Z. Petrov. Einstein Spaces, Pergamon, (1969).

[18] G.Fubini. Annali. di. Matematica. 8, (1903), 39.

[19] G.S. Hall and A.D. Rendall. Int. J. Theor. Phys. 28, (1989), 365.

[20] R. Shaw. Quart. J. Math. Oxford 21, (1970), 101.

[21] G.S. Hall Curvature Structure and Symmetries in General Relativity, World Scientific. To appear.

[22] L. Bianchi. Gen. Rel. Grav. 33, (2001), 2171.

[23] D. Montgomery and H. Samelson. Anns. Maths. 44, (1943), 454.

[24] A.D. Rendall. Class. Quan. Grav. 5, (1988), 695.

[25] G.S. Hall and M.S. Capocci. J. Math. Phys. 40, (1999), 1466.

[26] K. Nomizu. Anns Maths. 72, (1960), 105.

[27] G.S. Hall. Class. Quant. Grav. 6, (1989), 157.

\section{ACKNOWLEDGMENTS}

The author acknowledges valuable discussions with Dimitri Alexeevski, Bartolomé Coll, Michael Crabb and Alan Rendall and thanks M. Sharif for help in preparing the manuscript.

Department of Mathematical Sciences, University of Aberdeen, Aberdeen AB24 3Ue, SCOTLAND, UK. 